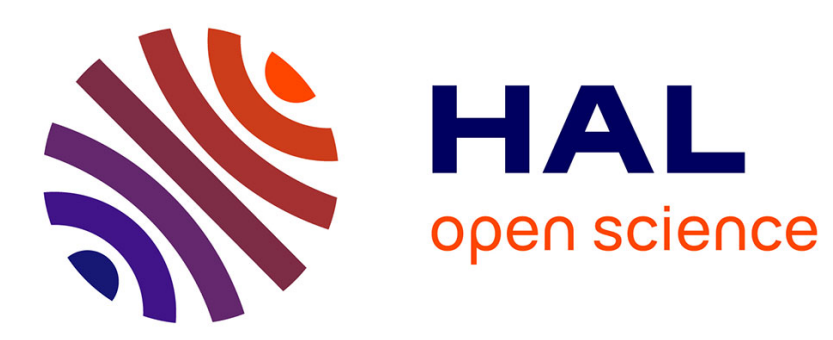

\title{
Anisotropy of the Meissner effect and the diamagnetic shielding in (TMTSF)2ClO4
}

\author{
D. Mailly, M. Ribault, K. Bechgaard, J.M. Fabre, L. Giral
}

\section{To cite this version:}

D. Mailly, M. Ribault, K. Bechgaard, J.M. Fabre, L. Giral. Anisotropy of the Meissner effect and the diamagnetic shielding in (TMTSF)2ClO4. Journal de Physique Lettres, 1982, 43 (20), pp.711-717. 10.1051/jphyslet:019820043020071100 . jpa-00232115

\section{HAL Id: jpa-00232115 https://hal.science/jpa-00232115}

Submitted on 1 Jan 1982

HAL is a multi-disciplinary open access archive for the deposit and dissemination of scientific research documents, whether they are published or not. The documents may come from teaching and research institutions in France or abroad, or from public or private research centers.
L'archive ouverte pluridisciplinaire HAL, est destinée au dépôt et à la diffusion de documents scientifiques de niveau recherche, publiés ou non, émanant des établissements d'enseignement et de recherche français ou étrangers, des laboratoires publics ou privés. 
Classification

Physics Abstracts

$74.30 \mathrm{C}-72.80 \mathrm{~L}$

\title{
Anisotropy of the Meissner effect and the diamagnetic shielding in $\left(\mathrm{TMTSF}_{2} \mathrm{ClO}_{4}\right.$
}

\author{
D. Mailly, M. Ribault (*) \\ Laboratoire de Physique des Solides (**), Université de Paris-Sud, Bât. 510, 91405 Orsay, France
}

K. Bechgaard

H.C. Oersted Institute, Universitetsparken 5, DK 2100, Copenhagen, Denmark

J. M. Fabre and L. Giral

Laboratoire de Chimie Organique et Structurale, U.S.T.L., 34060 Montpellier,-France

(Reçu le 21 juillet 1982, accepté le 6 septembre 1982)

\begin{abstract}
Résumé. - L'écrantage diamagnétique et l'effet Meissner mesurés sur un cristal de (TMTSF) ${ }_{2} \mathrm{ClO}_{4}$ sont fortement anisotropes. En très faibles champs magnétiques l'écrantage diamagnétique est complet pour chaque direction. Selon la direction a l'effet Meissner reste inférieur à $1 \%$ de sa valeur attendue alors que selon la direction $\mathrm{c}^{*}$, il atteint $80 \%$ de celle-ci. Les courbes d'aimantation en fonction du champ magnétique peuvent être interprétées par des effets de piégeage, les valeurs des champs critiques $H_{\mathrm{c}_{1}}$ sont donc surestimées. L'effet Meissner et le paramètre de Ginzburg-Landau pour la direction a montrent que cette direction est distincte.

Abstract. - The diamagnetic shielding and the Meissner effect measured on a single crystal of (TMTSF) ${ }_{2} \mathrm{ClO}_{4}$ are strongly anisotropic increasing from the $a$ to $b^{*}$ and $\mathrm{c}^{*}$ directions. In very low magnetic fields the diamagnetic shielding is complete for all directions. In the direction the Meissner effect never stands higher than $1 \%$ of its expected value whereas it reaches $80 \%$ in the $c^{*}$ direction. The aimantation curves versus magnetic field can be explained by pinning effects, but overestimated values of $H_{c_{1}}$ are obtained. The Meissner effect and the Ginzburg-Landau parameter for the a direction show that this direction is distinct.
\end{abstract}

1. Introduction - Superconductivity in an organic solid was first discovered in the material $(\mathrm{TMTSF})_{2} \mathrm{PF}_{6}[1,2,3]$, and has now been observed in several members of the (TMTSF) $2 \mathrm{X}$ family. The superconducting property has been shown to be a property of the bulk by A.C. susceptibility measurement [4], Meissner effect [5, 6] and most convincingly by recent specific heat measurements of the (TMTSF) ${ }_{2} \mathrm{ClO}_{4}$ [7].

(*) Work supported by ATP-C.N.R.S. program 1981.

(**) Laboratoire $\mathrm{n}^{\circ} 2$ associé au C.N.R.S. 
An important problem in evaluating these materials remains to be solved [8] : the crystal structure of this material gives a priori reason to expect strongly anisotropic properties because the material is composed of columnar stacks of TMTSF molecules separated by anion sheets. The tunnel [9], thermal conductivity [10] and magneto-absorption [11] measurements show important anisotropy effects near the superconducting temperature, while plasma energy [12] and magneto-transport properties [13] indicate significant interchain interactions.

In this report we address the anisotropies of diamagnetic shielding and Meissner effect in (TMTSF) ${ }_{2} \mathrm{CrO}_{4}$, so far the only material of this family where superconductivity occurs at ambient pressure below $1.2 \mathrm{~K}$. Similar work has been published [6] and prompts us to present our results since we observe major discrepancies, when the magnetic field is aligned along the a (stacking) axis.

We have used a SQUID technique to monitor the change of magnetization in the sample. The signal coils consist of two balanced coils, one containing the $\mathrm{ClO}_{4}$ sample and the other a tin calibration sample of similar shape. Two kinds of coils were made depending on the orientation of the sample to give the maximum filling factor. For the two perpendicular directions the sample coils are made of 20 turns of niobium wire, each allowing an internal section of $(4 \times 1) \mathrm{mm}^{2}$. For the parallel direction the sample coils are made of 100 turns of niobium wire, each giving an internal diameter of $1 \mathrm{~mm}$. The sample coils were surrounded by a polarizing coil giving a field up to $30 \mathrm{Oe}$. The coil system was placed in a lead shield filled with silicone oil to thermalize the sample and avoid vibration. The lead shield was thermalized to the mixing chamber of a dilution refrigerator giving temperature below $50 \mathrm{mK}$. A Permendur shield around the cryostat reduced the Earth's magnetic field to below $5 \mathrm{mOe}$. The remaining field can be further compensated by finding the current in the polarizing coil which minimized the Meissner signal of the Sn reference.

To calibrate the (TMTSF) ${ }_{2} \mathrm{ClO}_{4}$ signal we compare the $\mathrm{Sn}$ Meissner expulsion, assumed to be complete, and the (TMTSF $)_{2} \mathrm{ClO}_{4}$ signal in their volume ratio. The uncertainty of this measure is $10 \%$, due to the uncertainty of the volume estimates of the samples.

The demagnetization factor $D$ was determined by finding the ellipsoid which surrounded the most closely our sample. Initially the dimensions of the sample were $3.4 \times 0.7 \times 0.42 \mathrm{~mm}^{3}$ but when we changed the orientation from $\mathbf{b}^{*}$ to $\mathbf{c}^{*}$ it was " cleaved " along the a direction and its size was $3.4 \times 0.4 \times 0.42 \mathrm{~mm}^{3}$. Using the table given by Osborn [14] we find that $D_{a}=1$, $D_{b}=0.6, D_{c}=0.5$.

To orient the sample we first determined the $(0,0,1)$ face giving the $\mathbf{c}^{*}$ direction. The $\mathbf{b}^{*}$ direction was assumed to be perpendicular to the face oriented at approximately $96^{\circ}$ of the $(0,0,1)$ face. The a direction is taken along the needle axis. The alignment error is estimated to be inferior of the $5^{\circ}$ for parallel directions and inferior of $2^{\circ}$ for perpendicular directions.

The cooling time from nitrogen temperature to helium was around two hours, which corresponds to a " relaxed " sample [15]. This avoids random disorder effects, since it has been found that fast cooling of (TMTSF) ${ }_{2} \mathrm{ClO}_{4}$ suppresses the S.C. transition to $0.9 \mathrm{~K}$ and induces a SDW state just above $T_{\mathrm{c}}$.

2. Experimental results - Figure 1 exhibits a typical transition curve of the magnetization in a constant magnetic field. First the sample was cooled down to $50 \mathrm{mK}$ in zero field, then the magnetic field was applied and the decrease of the shielding signal observed upon warning. On the subsequent cooling the Meissner signal was monitored.

The transition temperature, $T_{\mathrm{c}}$, was determined in a field well below $H_{\mathrm{c}_{1}} . T_{\mathrm{c}}$ is defined at the intercept of the extrapolation of the linear part of the magnetization curve with $M=0$. We find $T_{\mathrm{c}}=1.12 \mathrm{~K}$. The first departure from $M=0$ occurs at $T=1.22 \mathrm{~K} . T_{\mathrm{c}}$ is independent of the field direction. The width of the magnetic transition $(0.5 \mathrm{~K})$ is about the same as the width of the resistive transition. 


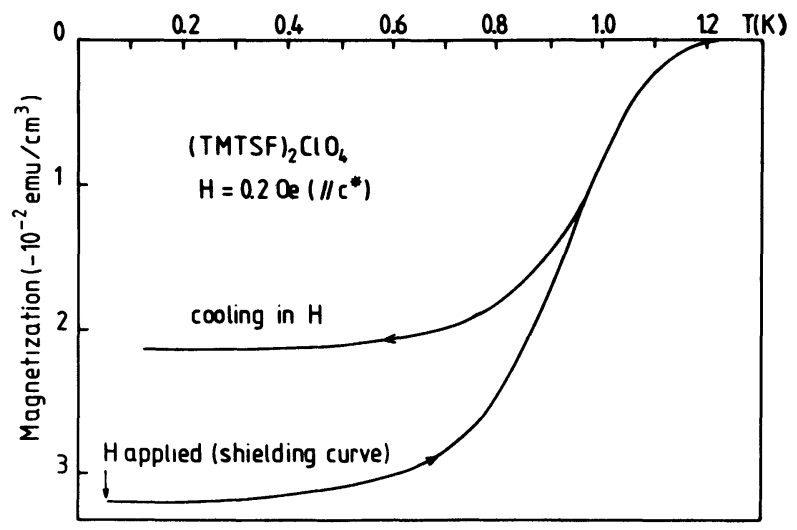

Fig. 1. - Diamagnetic shielding and Meissner effect of (TMTSF) ${ }_{2} \mathrm{ClO}_{4}$ versus temperature, for a magnetic field of 0.2 Oe oriented along the $c^{*}$ axis.

In the three dimensions the shielding signals are complete in the lowest field $(H=0.01 \mathrm{Oe})$ (as determined by the $\mathrm{Sn}$ reference).

The Meissner signal (determined by the ratio of the Meissner expulsion to the shielding signal in the same magnetic field) shows different behaviours depending on the direction of the magnetic field (see Fig. 2).

When the field is applied along the $c^{*}$ direction, the Meissner signal decreases slowly from $80 \%$ in the lowest field to $50 \%$ in $0.8 \mathrm{Oe}$.

Along the b* direction, the Meissner signal drops from $55 \%$ in $0.01 \mathrm{Oe}$ to $24 \%$ in $0.1 \mathrm{Oe}$ and decreases slowly below $15 \%$ in 0.8 Oe.

Along the a direction, the Meissner signal is around $1 \%$ from the lowest field, i.e. $H=0.01 \mathrm{Oe}$ to $H=0.8 \mathrm{Oe}$ (see table I).

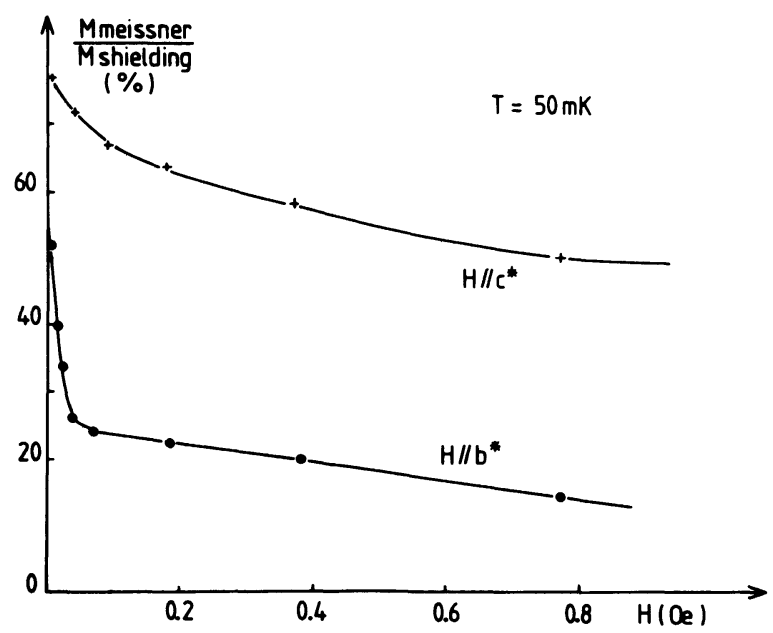

Fig. 2. - Meissner signal of (TMTSF $)_{2} \mathrm{ClO}_{4}$ versus applied magnetic field at $T=50 \mathrm{mK}$ for magnetic fields oriented along the $\mathbf{c}^{*}$ and $\mathbf{b}^{*}$ axes. 
In figure 3, the shielding magnetization is plotted versus the applied field for the three directions at $T=50 \mathrm{mK}$. The magnetization variation of the sample is the same either for a field variation at low temperature, or, in a given magnetic field, for the temperature variation up to the transition temperature.

The signal variation versus magnetic field is linear until an external field of 5 Oe for the $c^{*}$ direction, $0.4 \mathrm{Oe}$ for the $\mathrm{b}^{*}$ direction and $0.2 \mathrm{Oe}$ for the a direction. Taking this field to determine the value of $H_{\mathrm{c}_{1}}$ we find $H_{\mathrm{c}_{1 a}}=0.2 \mathrm{Oe},-H_{\mathrm{c}_{1 b}}=1.0 \mathrm{Oe}, H_{\mathrm{c}_{1 c}}=10 \mathrm{Oe}$ at $T=0.05 \mathrm{~K}$ (after corrections due to demagnetization effects).

Table I. - Meissner expulsion and shielding signal ratio for the three crystallographic axes.
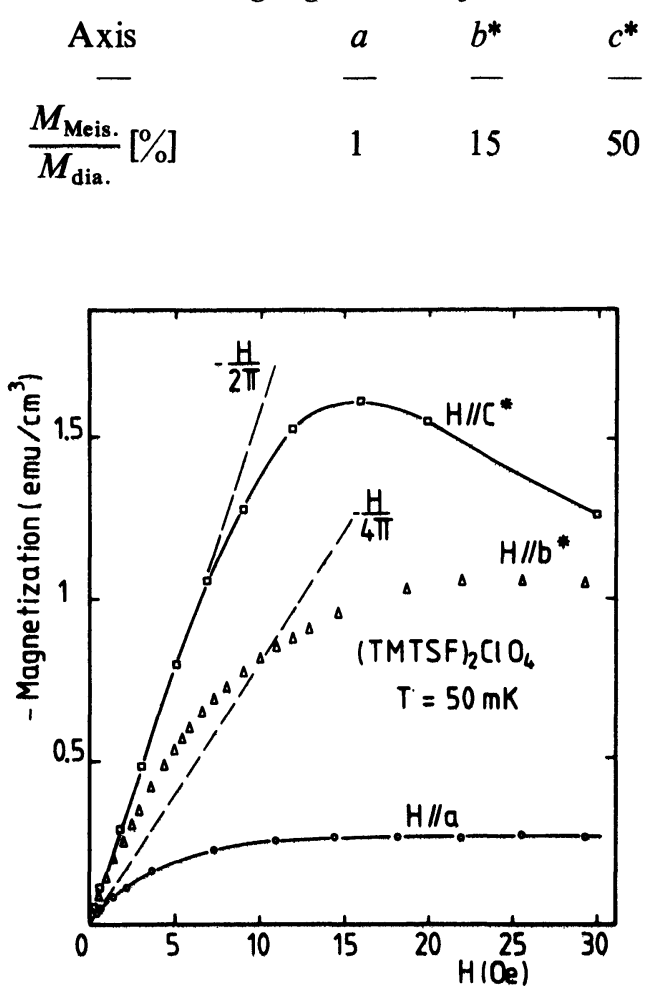

Fig. 3. - Diamagnetic shielding of (TMTSF) $)_{2} \mathrm{ClO}_{4}$ versus magnetic field at $T=50 \mathrm{mK}$, for magnetic fields oriented along the three crystallographic axes.

Figure 4 shows an hysteresis cycle performed along the $c^{*}$ direction at $50 \mathrm{mK}$. To establish this cycle the sample was first cooled in zero field down to $50 \mathrm{mK}$, then the magnetic field was applied step by step. The signal expulsion due to the $\mathrm{Sn}$ reference (known by making a run at $T=2 \mathrm{~K}$ ) was subtracted to give the hysteresis cycle.

3. Discussion - The shape of the variation of the magnetization versus the applied magnetic field, especially the curvature, can be analysed in two different ways : as a geometrical size effect [16] or as a current effect [17].

An experimental proof of the non occurrence of any size effects is given by the comparison of 


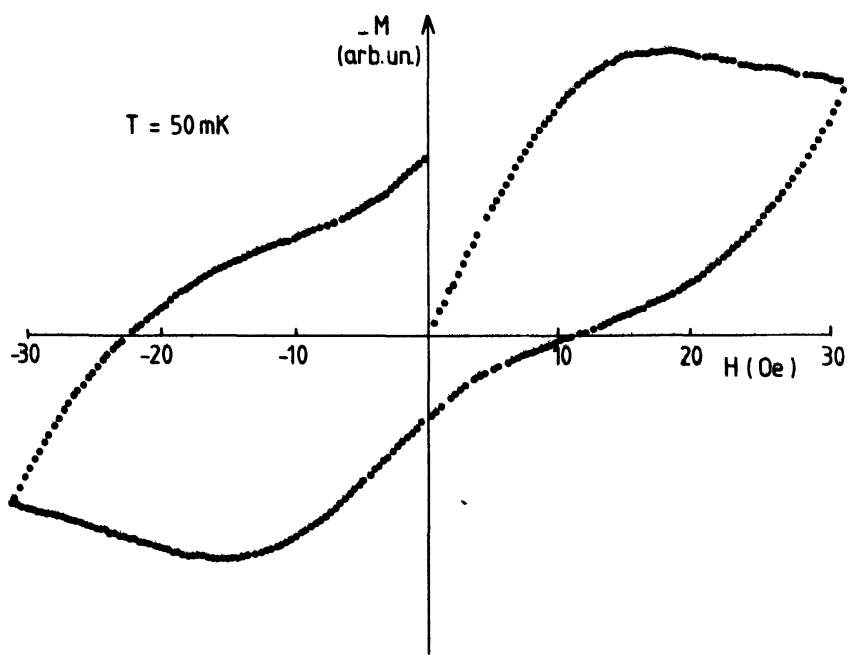

Fig. 4. - Hysteresis cycle of (TMTSF $)_{2} \mathrm{ClO}_{4}$ at $T=50 \mathrm{mK}$, with the magnetic field oriented along the $\mathrm{c}^{*}$ direction.

the initial susceptibility $\chi_{i}$ along the $\mathbf{b}^{*}, \mathbf{c}^{*}$ and a directions : when $\lambda_{\mathbf{j}} \sim L_{\mathbf{j}}\left(L_{\mathbf{j}}\right.$ : sample size, $\lambda_{\mathbf{j}}$ : penetration depth),

$$
\chi_{\mathrm{i}}=-\frac{1}{4 \pi}\left[1-\lambda_{\mathrm{j}} / L_{\mathrm{j}}\right]
$$

In the present results, $\chi_{\mathrm{i}}=-1 / 4 \pi$ within experimental uncertainties.

The curvature of the $M(H)$ curve cannot be understood as a geometrical size effect but has to be analysed through the shielding mechanism itself.

Such magnetization curves have been observed in filamentary superconductors where the Bean model gives a good description [17]. In this model the first departure from linearity of $M(H)$ is connected to the critical field of the bulk soft materials, whereas the saturation value of $M(H)$, connected to the critical currents of the hard material, varies with sample dimensions ; this is a good test for applicability. In the $\mathbf{a}$ and $\mathbf{b}^{*}$ directions the saturation is reached for magnetic fields values in a ratio inferior to 1.7 whereas the expected ratio is 8 ; the $\mathrm{c}^{*}$ curve does not have the main features of a Bean variation type : neither the relative position of the various regimes nor the values reached by $M(H)$ are in agreement with the model.

It is therefore not possible to give a good description of our measurements within a model of a net of filamentary conductors linked by localized bridges due to impurities or defects : the connection between longitudinal paths are of extended range type. The shape of the variation of $M(H)$ reflects the pinning of the supercurrents. This result is obvious for $\mathbf{a}$ and $\mathbf{b}^{*}$ curves and is confirmed for the $c^{*}$ curve by the hysteresis cycle (Fig. 4); the $c^{*}$ curve is not simply a type II superconductor curve but is strongly modified by the pinning, neither the maximum nor the first departure from linearity of $M(H)$ have a simple connection with $H_{c_{1}}$. The Meissner signal and the first magnetization are both measurements out of equilibrium. The Meissner effect when decreasing the temperature in a constant magnetic field is roughly equivalent to decreasing magnetic field at low temperature. The Meissner signal gives an underestimate and the first magnetization an overestimate of the critical fields. In the present case Meissner signals have never reached $100 \%$ and thus the underestimate is $H_{c_{1}}=0 \ldots$ and the overestimate is $H_{c_{1 a}}=0.2 \mathrm{Oe}, H_{\mathrm{c}_{1 b^{*}}}=$ $1.0 \mathrm{Oe}, H_{\mathrm{c}_{1 c^{*}}}=10 \mathrm{Oe}$. The values are in good agreement with those obtained by Schwenk et al. [6] when their perpendicular axis is the $c^{*}$ direction. 
With the $H_{\mathrm{c}_{1}}$ measurements and the value of $H_{\mathrm{c}}$ given by Garoche et al. [7] we can obtain the G.L. parameter $K_{3}$ by taking :

$$
H_{\mathrm{c}_{1}}=H_{\mathrm{c}} \frac{\log K_{3 \mathrm{i}}}{\sqrt{2} K_{3 \mathrm{i}}} \text { if } K_{3 \mathrm{i}}>10
$$

and the J. L. Harpen and V. Arp results [12] for $K_{3 i}<10$.

Table II shows the values obtained for the three crystallographic axes. The $K_{3 a}$ value is very high and indicates a very short mean free path for the electron in the $\mathbf{b}^{*}, \mathbf{c}^{*}$ planes.

We can go further in order to compare our results with previously published results by taking the Gorkov relation :

$$
H_{\mathrm{c}_{2 \mathrm{i}}}=\sqrt{2} K_{1 \mathrm{i}} H_{\mathrm{c}}
$$

where $K_{1 \mathrm{i}}$ is the G.L. parameter associated with $H_{\mathrm{c}_{2}} . K_{1}=K_{3}$ near $T_{\mathrm{c}}$. The difference between $K_{1}$ and $K_{3}$ " reflects the different sensitivity of the magnetic properties of the superconductor to the degree of non locality of the electrodynamics » [22]. Usually $K_{1}$ and $K_{3}$ differ by less than $25 \%[19]$.

Assuming $K_{1 \mathrm{i}}=K_{3 \mathrm{i}}$ we have estimated $H_{\mathrm{c}_{2 \mathrm{i}}}$ (see Table II). The values obtained for the b* and $\mathbf{c}^{*}$ directions are in good agreement with those found in the literature $[20,21,22]$. The $H_{\mathbf{c}_{2 a}}$ value is out of range, but some precautions are to be taken :

i) the orientation of the sample in the field is of importance. According to Turkevitch and Klemm [23] for a ratio of $M / m$ of the transverse to longitudinal effective mass of 50, an unalignment of $5^{\circ}$ gives a decrease of $H_{\mathrm{c}_{2}}$ by a factor higher than 2 ;

ii) the experimental values of $H_{\mathrm{c}_{2 a}}$ are still very controversial near $T_{\mathrm{c}}$ since the slope of $\mathrm{d} H_{\mathrm{c}_{2 a}} / \mathrm{d} T$ near $T_{\mathrm{c}}$ varies from $7.5 \mathrm{kOe} / \mathrm{K}$ to $70 \mathrm{kOe} / \mathrm{K}$ according to [20, 21, 22]. Furthermore most of these authors have taken, as a definition of $H_{\mathrm{c}_{2 a}}$, the magnetic field at half of the resistive transition versus magnetic field, instead of the $90 \%$ usually used. This definition modifies the $H_{\mathbf{c}_{2 a}}$ values by a factor of nearly 2 ;

iii) Greene et al. [20] have pointed out that the Pauli limit given in the BCS theory by $H_{\rho}=$ $18.6 T_{\mathrm{c}}$ will reduce the critical field to below $25 \mathrm{kOe}$ (for $T_{\mathrm{c}}=1.2 \mathrm{~K}$ ). But until now there is no experiment showing a curvature of the $H_{\mathrm{c}_{2 a}}(T)$ curve which indicates a Pauli type limitation.

The last point we want to emphasize is the very weak Meissner effect in the a direction, and infer that this has to be connected with the problem of moving the supercurrents in a plane where they are of the Josephson type; in the other planes, only one direction needs Josephson paths and a nearly ideal path is easier to find. The rapid increase of the Meissner signal in the $\mathbf{b}^{*}$ direction for $H<0.1 \mathrm{Oe}$ is likely of the same origin, both demonstrating the specificity of these materials where the magnetic field is not the only breaking mechanism; also in our opinion the intensity of the supercurrents itself is of importance.

Table II. - Critical fields and Ginzburg-Landau parameter for the 3 crystallographic axes. ( $H_{\mathrm{c}_{2}}$ is obtained from the Gorkov relations.)

\begin{tabular}{lccc}
\multicolumn{1}{c}{ Axis } & $a$ & $b^{*}$ & $c^{*}$ \\
- & - & - & - \\
$H_{\mathrm{c}_{1}} \mathrm{Oe}$ & 0.2 & 1.0 & 10.0 \\
$K_{3 \mathrm{i}}$ & 1020 & 157 & 9 \\
$H_{\mathrm{c}_{2}}[\mathrm{kOe}]$ & 63.5 & 9.77 & 0.56
\end{tabular}


4. Conclusion. - We have demonstrated the strong anisotropy of both Meissner and shielding signals. The shape of the variation of these signals versus the applied magnetic field can be reasonably explained as a flux pinning effects. This pinning cannot allow the determination of the first penetration magnetic field $H_{\mathrm{c}_{1}}$ but only its upper value. The stacking direction a appears to be distinct according to both the $H_{c_{1}}$ value and the Meissner effect. The extraordinary low Meissner signal will be interesting to study under pressure since the variation of the coupling should modify the behaviour. This work is in progress.

Acknowledgments. - We would like to thank D. Jérome, C. Bourbonnais and H. Raffy for many useful discussions. We are grateful for C. Godfrin for experimental advices.

\section{References}

[1] Jérome, D., Mazaud, A., Ribault, M. and Bechgaard, K., J. Physique-Lett. 41 (1980) L-95.

[2] Bechgaard, K., Carneiro, K., Rasmussen, F. B. and Jacobsen, C. S., Phys. Rev. Lett. 46 (1981) 852.

[3] Parkin, S. S. P., Ribault, M., Jérome, D. and Bechgaard, K., J. Phys. C 14 (1981) 5305.

[4] Ribault, M., Benedek, G., Jérome, D. and Bechgaard, K., J. Physique-Lett. 41 (1980) L-397.

[5] Andres, K., Wudl, F., McWhan, D. B., Thomas, G. A., Nalewajek, D. and Stevens, A. L., Phys. Rev. Lett. 45 (1980) 1449.

[6] Schwenk, H., Neumaier, K., Andres, K., Wudl, F., Aharon-Shalom, E., Mol. Cryst. Liq. Cryst. 79 (1982) 277.

[7] Garoche, P., Brusetti, R., Jérome, D. and Bechgaard, K., J. Physique-Lett. 43 (1982) L-147.

[8] JÉrome, D., Mol. Cryst. Liq. Cryst. 79 (1982) 155.

[9] More, C., Roger, G., Sorbier, J. P., Jérome, D., Ribault, M. and Bechgaard, K., J. PhysiqueLett. 42 (1981) L-313.

[10] DJuREK, D., to be published (1982).

[11] Ng, H. K., Timusk, T., Delrieu, J. M., Jérome, D., Bechgaard, K. and Fabre, J. M., J. PhysiqueLett., to be published (1982).

[12] Jacobsen, C. S., TANner, D. B., BechgaArd, K., Phys. Rev. Lett. 46 (1982) 1142.

[13] Chaikin, P. M., Haen, P., Engler, E. M. and Greene, R. L., Phys. Rev. B., to be published.

[14] Osborn, J. A., Phys. Rev. 67 (1945) 351.

[15] Garoche, P., Brusetti, R. and BechgaARd, K., to be published (1982).

[16] Lock, J. M., Proc. R. Soc. A 208 (1951) 391.

[17] Bean, G., Rev. Mod. Phys. 36 (1964) 31.

[18] Harden, J. L., Arp, V., Cryogenics 3 (1963) 105.

[19] Eilenberger, G., Phys. Rev. 153 (1967) 584.

[20] Greene, R. L., Haen, P., Huang, S. Z., Engler, E. M., Choi, M. Y. and Chaikin, P. M., Mol. Cryst. Liq. Cryst. 79 (1982) 183.

[21] Murata, K., Anzai, H., Kajimura, K., Ishiguro, T. and Saito, G., Mol. Cryst. Liq. Cryst. 79 (1982) 283.

[22] Gubser, D. U., Fuller, W. W., Poehler, T. O., Stokes, J., Cowan, D. O., Lee, M. and Bloch, A. N., Mol. Cryst. Liq. Cryst. 79 (1982) 225.

[23] Turkevitch, L. A., Klemm, R. A., Phys. Rev. B 19 (1979) 2520. 